\title{
An exploratory trial of the effectiveness of an enhanced consultative approach to delivering speech and language intervention in schools
}

International Journal of Language and Communication Disorders (in press)

\section{Carol Mecrow \\ Jennie Beckwith}

Northumberland County Council Communication Support Service

Till House, Hepscott Park

Stannington

Morpeth NE61 6NF

England, UK

Tel: 01670534344

Fax: 01670534343

Email: Carol.Mecrow@northumberland.gov.uk; Jennie.Beckwith@ northumberland.gov.uk

\section{Thomas Klee}

School of Education, Communication \& Language Sciences and Institute of Health \& Society King George VI Building

Newcastle University

Newcastle upon Tyne NE1 7RU

England, UK

Tel: 01912227452

Fax: 01912226518

Email: thomas.klee@newcastle.ac.uk

Keywords: developmental language disorder, developmental speech disorder, intervention, treatment effectiveness, consultative model; exploratory trial 


\begin{abstract}
Background: Increased demand for access to specialist services for providing support to children with speech, language and communication needs prompted a local service review of how to best allocate limited resources. This study arose as a consequence of wishing to evaluate the effectiveness of an enhanced consultative approach to delivering speech and language intervention in local schools.

Aims: The purpose of this study was to evaluate an intensive speech and language intervention for children in mainstream schools, delivered by specialist teaching assistants. Methods \& Procedures: A within-subjects, quasi-experimental exploratory trial was conducted, with each child serving as his or her own control with respect to the primary outcome measure. Thirty-five children between the ages of 4;2 and 6;10 (years; months) received speech and/or language intervention for an average of four one-hour sessions per week over 10 weeks. The primary outcome measure consisted of change between pre- and post-intervention scores on probe tasks of treated and untreated behaviours summed across the group of children, and maintenance probes of treated behaviours. Secondary outcome measures included standardized tests (Clinical Evaluation of Language Fundamentals Preschool $^{U K}$; Diagnostic Evaluation of Articulation and Phonology) and questionnaires completed by parents/carers and school staff before and after the intervention period. Outcome \& Results: The primary outcome measure showed improvement over the intervention period, with target behaviours showing a significantly larger increase than control behaviours. The gains made on the target behaviours as a result of intervention were sustained when re-assessed 3-12 months later. These findings were replicated on a second set of targets and controls. Significant gains were also observed on CELF-Preschool ${ }^{\mathrm{UK}}$ receptive and expressive language standard scores from pre- to post-intervention. However, DEAP standard scores of speech ability did not increase over the intervention period, although
\end{abstract}


improvements in raw scores were observed. Questionnaires completed before and after intervention showed some significant differences relating to how much the child's speech and language difficulties affected him/her at home and at school.

Conclusions: This exploratory study demonstrates the benefit of an intensive therapy, delivered by specialist teaching assistants for remediating speech and language difficulties experienced by young children in mainstream schools. The service delivery model was perceived by professionals as offering an inclusive and effective practice and provides empirical support for using both direct and indirect intervention in the school setting.

\section{What this paper adds}

What is already known on this subject

Debate surrounds the merits of different approaches to delivering speech and language intervention to children, with a growing reliance upon a consultative role played by Speech and Language Therapists in school settings. However, little research exists documenting the effectiveness of consultative approaches.

What this study adds

Intensive intervention, comprised of direct and indirect support and delivered by specialist teaching assistants, was shown to be effective at improving children's language performance. Parents and school staff were positive about the benefits of this approach to delivering speech and language intervention. 


\section{Background}

This study examines the effectiveness of an ongoing speech and language intervention programme for school-age children in the North East of England. The intervention was delivered by specialist teaching assistants ${ }^{1}$ (STA) under the direction of speech and language therapists (SLT) having a dual qualification in teaching and by specialist teachers ${ }^{2}$. The main objective of the study was to examine whether an existing, enhanced consultative approach to delivering intervention under real-world working conditions was effective in bringing about change in specific linguistic behaviours targeted for intervention in relation to a set of untreated linguistic behaviours. The secondary objectives of the study were to examine the extent to which children's performance on standardized speech and language tests changed, and whether the views of parents and school staff changed over the course of the intervention period.

Supporting children and young people in need in schools has encouraged multiprofessional working with teachers, SLTs and others sharing knowledge and practice for the benefit of the child. Such an approach was promoted by government proposals to establish Children's Trusts in the UK following the White Paper Every Child Matters (Department for Education and Skills 2003). Building provision around the needs of children with integrated services and multi-disciplinary teams is currently a primary aim of government, both nationally and locally.

One of the recommendations of the recent Bercow Report is “...to develop effective collaborative practice between different services and members of the workforce" (Department for Children, Schools and Families 2008: 9). The Bercow Report was informed by research which sought to explore the effective and efficient use of services for children with speech, language and communication difficulties (Lindsay et al. 2008). It considered service delivery in six local authorities and primary care trusts in the UK, identifying 
variation in current practice and providing recommendations for the future development and evaluation of children's services. Collaborative working and the development of integrated services was encouraged, as was a consideration of working practices to include, for example, comparing the use of a consultative model with direct teaching/therapy. The intervention model employed in the current study reflects one example of such collaboration between education and SLT provision for children.

\section{Consultative Model of Service Delivery}

Historically, speech and language intervention for children in the UK was provided in community clinics by SLTs working directly with children for a time-limited block of intervention (e.g., see Glogowska et al. 2000). In commenting about the effectiveness of such an approach, Law and Conti-Ramsden (2000: 909) concluded that “...offering limited amounts of speech and language therapy [was] not a tenable solution to the problem”. Increasingly, SLTs in the UK are working in schools using an indirect model of service delivery, often referred to by service providers as consultative (Law et al. 2002). In this approach, the SLT assesses a child and plans a programme of intervention which is then usually delivered by a member of the school staff. This model recognises the importance of the social and academic settings in which children learn and takes advantage of those settings in the delivery of intervention. In reviewing the consultative model of delivering speech and language services, Law et al. (2002) discussed the prerequisites for successful practice. These include (1) training and availability of staff to deliver input, especially assistants within school; (2) time and availability of staff with whom to consult; (3) understanding and knowledge of the classroom environment and curriculum expectations; (4) parental support; and (5) sufficient trained staff to offer consultation.

In practice, interpretation and application of the consultative model is not always consistent. Direct contact time between the child and therapist is flexible and targets may be 
worked on within or outside the classroom. Furthermore, therapy may be delivered individually or in groups and be of varying duration, both in terms of the length and number of sessions offered. In summary, the definition of the term consultancy is far from clear and requires further debate and clarification (Lindsay et al. 2002, Gascoigne 2006).

\section{The Evidence Base for Language Intervention with Children}

Summaries of evidence regarding the efficacy and/or effectiveness of intervention for children with spoken speech and language difficulties have been presented in two recent systematic reviews. Law et al. 2004 presented a statistical summary (meta-analysis) of intervention studies related primarily to pre-school children, while Cirrin and Gillam (2008) reviewed studies published between 1985 and 2005 related to school-age children. The authors of both reviews concluded that while there was some evidence for the positive effects of such interventions, further research was necessary to close the gaps in what is currently known about what works with which children. Among other things, Cirrin and Gillam suggested that further research was needed "on the effectiveness of classroom-based and collaborative language interventions...collaborative consultation...classroom versus individual treatment for language disorders...and integrated indirect services...”(p. S132).

A recent randomised controlled trial (RCT) in Scotland examined four modes of delivering school-based language intervention to children aged 6 to 11 with primary language impairment (Boyle et al. 2007). Children in each of the four experimental groups received intervention either directly (by an SLT) or indirectly (by a Speech and Language Therapy Assistant; SLTA) in either individual or group settings (Boyle et al. 2007), usually by removing the child from the classroom to conduct the intervention (McCartney et al. in press). Intervention was carried out using a specially written manual of language-learning activities and was delivered three times per week for 15 weeks, for an average of 38 sessions per child. A fifth group of children who received existing levels of community-based therapy, 
thought to be based on consultancy approaches (McCartney et al. in press), served as a control group. The groups were statistically similar on the primary outcome measure, a standardized test of receptive and expressive language, before the intervention commenced. At the end of the intervention period, all four groups had significantly higher test scores than the control group but did not differ among themselves. That is, indirect therapy was as efficacious as direct therapy and group therapy was as efficacious as individual therapy using this intervention programme. Significant language test score gains were reported for children with expressive language impairment but not for children with mixed receptive-expressive impairments.

McCartney et al. (in press) summarised the results of a cohort study of 38 children that employed school staff to deliver the same manualised therapy as was used in the Boyle et al. (2007) trial. The intervention was delivered under the guidance of an SLT over a similar period of time (four months) for an average of 26 sessions. However, the intervention as delivered by educational staff did not result in significant improvements in standardized language test scores when compared to the control group of the Boyle et al. study. The difference in outcomes between the cohort study and the RCT could have been due to any number of different factors, including personnel factors or the amount of therapy delivered.

\section{Enhanced Consultative Model}

In the North East of England, a specialist team is funded through education to work with children in mainstream schools who have speech, language and communication needs. As part of the provision offered to schools, a team of STAs are employed to work under the guidance of a specialist teacher or teacher/therapist and made available to schools to work intensively with children. The approach represents an enhanced consultative model employing the principles outlined by Law et al. (2002) but with the following additional features: 
1. Collaboration is explicit at the outset of and during intervention. Staff from school and the specialist service meet with parents/carers before input and targets are set following assessment, observation and consideration of current curricular objectives. A contract is agreed between the specialist team and the school, designed to ensure that a member of the school staff is able to continue the programme of intervention once STA intensive input has ended.

2. STAs work closely with Learning Support Assistants (LSAs) in school, providing training for them to continue implementing the child's programme.

3. Communication between home, school and the specialist team is maintained throughout the intervention with daily opportunity for the STA to liaise with school staff (including class teacher, LSA and the wider school community), parents and carers through the use of a home/school diary, and the specialist team through continuous access with shared accommodation and formal and informal meetings.

4. Support for the child takes the form of daily teaching sessions led by the STA, who has both specialist knowledge and experience of working with children in school.

5. Weekly monitoring of progression against targets is supplied to schools and feedback is given to parents in the diary.

6. Targets are revised as required during the input period.

7. Once the STA has withdrawn from the school, the child's progress and the school's input continues to be monitored through visits during the following term. Thereafter, ongoing support is provided by the STA, specialist teacher or teacher/therapist as appropriate. 


\begin{abstract}
Aims
This study investigated the effectiveness of an ongoing, intensive intervention programme designed by specialist teachers and teacher/therapists and delivered by specialist teaching assistants working with children experiencing speech and/or language difficulties. The primary outcome measure was change on probe tasks designed to measure specific intervention goals and taken immediately before and after the intervention period and again several months later. Secondary outcome measures included standardized speech and language tests and questionnaires completed by school and parents, administered before and after the intervention period. Taken together, these measures were intended to reflect the impact of the intervention with respect to (1) targeted (versus control) linguistic behaviours, (2) standardized, norm-referenced speech and language performance and (3) perception of parents and school staff regarding the impact of the intervention on the child's home and school life. The study was conducted under conditions which reflected the real-world working practices of those involved. The motivation for conducting the study was to provide information about an existing model of service delivery that could be used to inform future service planning.
\end{abstract}

\title{
Methods and procedures
}

\section{Participants}

The research procedures reported in this investigation were approved by the Speech and Language Sciences Research Ethics Committee of the School of Education, Communication and Language Sciences at Newcastle University. Informed written consent was given by the parent or carer of each participating child. Although school staff were aware of the study taking place, this was not discussed with the children since the intervention they received would have taken place anyway. 
Children for the study were selected from among 113 referrals made to the specialist service between September 2004 and December 2006. Children presenting with any of the following were excluded from the study: (1) diagnosis of autism spectrum disorder; (2) parent or teacher reported hearing loss; (3) dysfluency; (4) English as an additional language; (5) geographical considerations; or (6) language difficulties not considered severe enough to warrant intensive specialist service. Children not receiving input from the STA received less intensive support from a specialist teacher/therapist.

Thirty-five children (27 boys, 8 girls) with an identified speech and/or language need took part in the study. They ranged in age from 4 years 2 months to 6 years 10 months $(M=$ 60.3 months), attended mainstream primary schools and were identified as at School Action Plus (Department for Education and Skills 2001) ${ }^{3}$. They were drawn from 30 urban and rural schools across a large geographical area. The average $\mathrm{IDAC}^{4}$ score of the schools $(\mathrm{M}=0.21$, $\mathrm{SD}=0.12)$ was similar to that of the national average $(0.20)$. Children were referred to the specialist service either by school personnel or the local SLT department because of concerns about their speech and language skills. In addition to professional concern being registered, all participating children scored 1.5 standard deviations or more below the mean on either the receptive or expressive subscale of the Preschool Language Scale-3 UK (PLS-3UK;

Zimmerman et al. 1997) or the Percent Phonemes Correct score of the Diagnostic Evaluation of Articulation and Phonology (DEAP; Dodd et al. 2002). For descriptive purposes the Developmental Test of Visual-Motor Integration (VMI; Berry and Buktenica 1997) was also administered. Children's performance on the standardized tests is summarised in Table 1. Within these general parameters, the children differed in their profiles of speech and language abilities. With respect to performance on the PLS-3UK and DEAP, 14 children demonstrated receptive and expressive language difficulties; seven had speech, receptive and expressive language difficulties; five had speech and expressive language difficulties; three 
had speech difficulties only; and one had speech and receptive language difficulties. In the case of five others, although no professional concern was registered regarding their receptive language, difficulty in this area could not be ruled out since the PLS receptive subscale was not administered.

\section{[Table 1 about here]}

\section{Procedures}

The intervention programme for each child commenced upon completion of the selection procedures outlined above. An Individual Education Plan (IEP) was drawn up for each child that involved selecting goals directed at developing one or more of the following areas: attention and listening, auditory memory, receptive and expressive language, phonological awareness, literacy and all aspects of speech. From these, two intervention targets and two controls were identified for each child for the purpose of the study, allowing change over the course of the intervention programme to be tracked. Target behaviours were matched to control behaviours on the basis of general developmental characteristics taken from standard textbooks and assessments (Boehm 2001, Owens 2001, Dodd et al. 2002). For example, language targets such as "in front of" and "behind" were matched to controls such as "next to" and speech targets such as voicing were matched to controls such as final consonant deletion. Intervention targets and control behaviours were not randomly assigned but were selected to equate to each other in terms of developmental level wherever possible. Appendix A lists the targets and controls for each child.

Each child received input from one of five STAs, delivered under the guidance of an SLT holding a teaching qualification or a specialist teacher. The intervention planned was discussed before input began in school with relevant staff and parents/carers.

Children participated in an average of 39 sessions $(\mathrm{SD}=11.44)$ over the course of the intervention period, each lasting between 45-60 minutes. Sessions took place in the child's 
school an average of four times per week over a 10 week period. The usual duration of support from the STA was one school term, although for some children a second term of support was necessary. This decision was based on the needs of individual children.

On average, each child's IEP contained 14 targets (range 6-24). From these, two target and two control behaviours were identified in order that they could be measured for the purpose of this study. These particular behaviours are specified in Appendix A and further explained below (see Probe task section). Each intervention session typically focussed on six targets. It was neither possible nor appropriate to work on every IEP target in every session. However, all targets were addressed each week and every session included at least one treatment target.

The teaching methods used varied according to the child's specific IEP targets and combined commercially available programmes with resources and activities created by the STAs. Teaching related to the treatment targets was delivered individually, although group work was sometimes undertaken for other IEP targets if deemed appropriate. Although the sessions themselves varied, they all included motivational games and activities which provided opportunities for practice and repetition. A typical activity to teach comprehension of prepositions, for example, would involve the STA placing toys around the room and modelling the target form. Subsequently, pictorial representations of the same concept, depicting a different set of objects, would be introduced. Any opportunities which occurred during the teaching sessions to reinforce the child's understanding of the targets would be utilised. Wherever possible, teaching was undertaken in quiet environments, free of distraction.

\section{Outcome measures}

Change in children's speech and language ability over the course of the intervention period was examined with a set of primary and secondary outcome measures. The primary outcome 
measure consisted of change over time on a probe task designed to track children's performance on two specific target and control behaviours, while secondary measures consisted of a standardized test of speech or language ability and questionnaires administered to parents or carers and the children's classroom teachers. These are described below.

\section{Probe task}

Individual 10-item probe tasks were designed to measure the accuracy of two specific target and control behaviours for each child at regular intervals before, during and after the period of intervention on which they were worked. This is referred to hence as the treatment phase and relates only to that portion of the broader intervention period which focused on these specific behaviours. The target, but not control, behaviours were also probed again after the intervention period ended in order to examine whether the behaviours targeted in therapy had been maintained over time. Target and control probes were individually selected from each child's IEP and tested by randomly selecting 10 cards from a pool of 15 (e.g. pictures of objects designed to elicit word-initial /s/ or subject pronouns) on each assessment occasion by the STA. For most children, the probe tasks were administered on six occasions (range 4-6) before the treatment phase, in order to establish a pre-treatment baseline for the two targets that were part of the focus of the subsequent intervention, and the two controls which were not. During this pre-treatment, baseline phase of the study, neither the target nor control behaviours were taught; however, other goals from the IEP were worked on during this time. During the treatment phase, probe tasks were administered on an average of six more occasions (range 3-8) in order to track change in target and control behaviours over the course of the intervention. During the post-treatment phase, an average of six further probes were administered in successive sessions to establish the status of the target and control behaviours in the absence of intervention (range 3-6). Finally, a single maintenance probe was administered 3-12 months after the intervention period ended in order to examine 
whether the two target behaviours had been maintained in the absence of continued support. The timing of this last probe varied across the children since the decision to examine maintenance was not made until after the intervention ended for some children. Control behaviours were not re-tested at this point, since they often became the next target for intervention.

\section{Standardized test}

A standardized test of children's receptive and expressive language, the Clinical Evaluation of Language Fundamentals - Preschool (UK) (CELF-P ${ }^{\mathrm{UK}}$; Wiig et al. 2000), was administered before and after the intervention period to determine whether localized intervention effects would generalize to a more global measure of language ability. The CELF-P $\mathrm{P}^{\mathrm{UK}}$ consists of three receptive and three expressive subtests and from these, composite scores of receptive and expressive language ability are derived. The Diagnostic Evaluation of Articulation and Phonology (DEAP; Dodd et al. 2002) was also administered, but only to children deemed to have a speech disorder. The standardized tests were considered to be secondary outcome measures since, if any gains on the IEP targets were made as a result of intervention, the probe task was more likely to be sensitive to them than tests of broader abilities. Moreover, norm-referenced tests by their nature may not be sensitive to registering small, but real, gains resulting from intervention.

\section{Questionnaires}

Parents and school staff were asked to complete questionnaires for each child on two occasions. A questionnaire was given to either one parent or carer of each child immediately before the intervention period and again immediately after. Similarly, school staff were also asked to complete a questionnaire immediately before and after the intervention period. The questions asked of each respondent are listed in Appendix B. Each question was accompanied by a closed set of response categories, some which were dichotomous (i.e., yes, no) and 
others which had multiple response options. An example of the latter may be seen in question, Do you think the pupil's speech and language difficulties affect him/her at school?, which had the following options: (1) Very little, on odd occasions; (2) Sometimes, some of the time; (3) Often, much of the time; and (4) A lot, most of the time.

Since not all items on the questionnaires were answered by every respondent, statistical analyses were conducted only on those items for which responses were given on both the pre- and post-intervention questionnaires. The purpose of administering the questionnaires was to explore teachers' and parents' (or, in some cases, carers') subjective impressions of the difficulties the children were experiencing, the role the intervention service played in the school, and the perceived progress of the children over the intervention period. Like the standardized test, the questionnaires served as a secondary outcome measure.

\section{Outcomes and results}

Pre- and post-treatment outcomes are presented for the probe task, the standardized language test, and the parent and teacher questionnaires.

\section{Probe task}

Separate $2 \times 2$ repeated measures analysis of variance procedures were conducted to evaluate the effect of the intervention. One analysis focused on the first intervention target selected for each child, compared with its control. The other analysis focused on the second intervention target compared with its control. The two intervention/control conditions were not combined in the statistical analysis since they were administered sequentially. The dependent variable was computed as the number of correct productions on a probe task consisting of 10 items randomly chosen from a pool of 15 items. The within-subjects factors were Target with two levels (intervention and control) and Time with two levels (pre- and post-treatment). The 
Target and Time main effects and the Target $\mathrm{x}$ Time interaction were tested using the multivariate criterion of Wilks' lambda $(\Lambda)$. Figure 1 presents a graph depicting the mean number of items correct across all children for each session the probe task was given. Table 2 presents summary statistics for each probe condition collapsed across the sessions in each phase.

[Figure 1 about here]

[Table 2 about here]

In the first ANOVA, the Target main effect was significant, $\Lambda=.12, F(1,34)=$ $250.84, p<.0005$, partial eta squared $=.88$, as were the Time main effect, $\Lambda=.09, F(1,34)$ $=349.24, p<.0005$, partial eta squared $=.91$ and the Target $\mathrm{x}$ Time interaction, $\Lambda=.14, F(1$, $34)=204.32, p<.0005$, partial eta squared $=.86$. Four paired-samples $t$ tests were conducted to follow up the significant interaction. Each of these was significant, controlling for familywise error rate across the four tests at the .05 level using the Holm's sequential Bonferroni procedure. While both targets and controls showed significant gains over the treatment phase, the average increase for targets was substantially larger than the gain for controls (8.75 compared to 1.38 items out of 10 respectively), with this gain likely to be the result of the intervention. Children's gains on the treatment targets over the course of the intervention period represented large effect sizes ${ }^{5}$.

These results were replicated with the second set of target/controls, based on 33 children (data were unavailable for two of the 35 children). In the second ANOVA, the Target main effect was significant, $\Lambda=.21, F(1,32)=117.49, p<.0005$, partial eta squared $=.79$, as were the Time main effect $, \Lambda=.19, F(1,32)=135.91, p<.0005$, partial eta squared $=.81$ and the Target $\mathrm{x}$ Time interaction, $\Lambda=.23, F(1,32)=107.35, p<.0005$, partial eta squared $=.77$. As in the previous analysis, paired-samples $t$ tests were conducted to follow up the significant interaction and, as previously, each was significant. While both 
targets and controls showed significant gains over the treatment phase, the average increase for targets was larger than that of controls (7.43 compared to 1.07 items out of 10 respectively). As was the case with the first set of target and controls, this gain was likely to be due to the intervention and the effect sizes were considered to be large.

The sustainability of gains in the target behaviours noted at post-treatment was examined by testing whether children's performance on the probe task showed any decrease between the post-treatment and maintenance periods. Paired $t$-tests were used to examine this, since the maintenance condition could not be incorporated into the ANOVAs reported above, as only the probe task for targets was administered during the maintenance period. Children's performance on the probe tasks conducted during the maintenance period was similar to what it was at the end of the intervention period. For the first target, children's score on the probe task during the maintenance period $(\mathrm{M}=8.63, \mathrm{SD}=3.19)$ was similar to their performance during the post-treatment period $(\mathrm{M}=9.11, \mathrm{SD}=2.20), t(29)=-0.83, p=.42$. Similarly, for the second target children's score on the probe task during the maintenance period $(\mathrm{M}=8.64$, $\mathrm{SD}=3.30)$ was similar to their performance during the post-treatment period $(\mathrm{M}=8.36, \mathrm{SD}$ $=2.76), t(27)=0.48, p=.63$.

Since the study was designed to test the effectiveness of an enhanced consultative model of service delivery with a rather heterogeneous group of children representing a typical caseload, all statistical analyses were conducted on the group as a whole. While it is possible that the intervention may have differentially affected children having various profiles of language ability, as has been shown in previous studies (e.g., Boyle et al. 2007), it was not possible to conduct sub-group analyses due to the small sample size of the sub-groups.

\section{Standardized tests}

Unlike the probe task, it was not possible to determine whether changes in language and speech ability, as measured by the CELF-P $\mathrm{P}^{\mathrm{UK}}$ and the DEAP respectively, were due 
specifically to the intervention or to some other factor such as general maturation or classroom learning. As was expected, there was a statistically significant increase in children's raw scores over the course of the intervention period on each subtest of the CELF$\mathrm{P}^{\mathrm{UK}}$, as well as on the receptive and expressive composite scales. This might have been anticipated even in the absence of an intervention effect. However, we also examined standard score differences, reasoning that significant improvements in these would probably only occur if changes beyond what would have been expected by maturation, and possibly attributable to intervention, had taken place. Table 3 presents the group's performance, in terms of standard scores, on the standardized tests administered immediately before and after the intervention period. The children made significant standard score gains on both the receptive and expressive composite scales of the CELF-P ${ }^{\mathrm{UK}}$, representing small ${ }^{5}$ effect sizes (partial eta square $=.12$ and .08 respectively). Significant gains were also observed on three of the six subscales, with the Sentence Structure subscale showing a large effect size (.34) and the Formulating Labels and Word Structure subscales showing small effect sizes (.10 and .11 respectively) . The standard score increase observed on the DEAP Percent Phonemes Correct was not statistically significant.

[Table 3 about here]

\section{Questionnaires}

Differences between pre- and post-intervention responses were analysed using the Wilcoxon signed-rank test (for items having multiple response categories) or McNemar's test (for items having dichotomous response categories). All tests were one-tailed since we anticipated that respondents would generally report improvement in response to the intervention; probability levels were not adjusted since the questionnaire was exploratory.

Parent questionnaires. Thirty-three of 35 parents returned questionnaires given to them before the intervention period, while 26 returned questionnaires given to them after. No 
statistically significant differences were noted between the pre- and post-treatment questionnaire items except in two cases (see Appendix B). Ratings for Question 1were significantly lower after the intervention period $(z=-2.84, p<.002)$. The same held for Question $2(\mathrm{z}=-2.31, \mathrm{p}=.01)$, indicating that parents felt their children were less affected by their speech and language difficulties at home and at school after, as compared to before, the intervention period.

School questionnaires. Thirty-three of 35 school staff returned pre-intervention questionnaires and 31 returned post-intervention questionnaires. Statistically significant differences were noted on 10 of the 20 questionnaire items. Like parents, school staff also reported that the children were less affected by their speech and language difficulties at school after the intervention period as compared to before $(z=-3.63, p<.0005)$. Staff also reported having concerns about fewer pupils' progress after the intervention as compared to before $(\mathrm{z}=-3.00, \mathrm{p}<.002)$ and having concerns about fewer children's future progress after the intervention $(\mathrm{z}=-3.00, \mathrm{p}<.002)$. Staff reported an improvement in children's listening skills $(\mathrm{z}=-1.94, \mathrm{p}<.04)$, as well as with the children's concentration and attention to tasks ( $\mathrm{z}$ $=-2.83, \mathrm{p}<.003)$. Finally, staff noted improved academic attainment in the areas of reading $(\mathrm{z}=-2.71, \mathrm{p}<.004)$, letter formation $(\mathrm{z}=-2.54, \mathrm{p}<.007)$, sound-letter awareness $(\mathrm{z}=-2.75$, $\mathrm{p}<.003)$, writing words $(\mathrm{z}=-2.48, \mathrm{p}<.006)$ and spelling $(\mathrm{z}=-2.07, \mathrm{p}<.031)$. The items that did not show change over the course of the intervention are noted in Appendix B.

\section{Conclusions and implications}

Statistical analysis of the probe measures indicated that children's performance on treated linguistic structures showed a large and significant improvement following intervention compared to their performance on untreated linguistic structures (see Figure 1 and Table 3). Furthermore, this improvement was maintained following withdrawal of 
specialist intervention. Children's performance on the control measures increased significantly but to a much smaller extent, relative to their performance on the target measures. It may be that children's scores on the probe tasks improved over time as a result of repeated testing. However, although performance on both target and control measures was assessed in the same way, gains made on target behaviours were substantially greater than those made on control behaviours in every case, suggesting that the greater gains made on target behaviours were likely to be the result of intervention. The fact that gains on target behaviours were maintained several months after the intervention is also of interest in that these behaviours were not specifically targeted in school after withdrawal of specialist intensive support, which suggests that children had mastered the targeted structures, at least as measured by the probe task. Although children's improvement on the probe task could be considered to represent a real and clinically important change with respect to the target behaviours, further research is needed to determine whether the change demonstrated on this task generalised to other contexts such as conversation and narration.

Significant improvements in standard scores on a test of general receptive and expressive language abilities (CELF-P ${ }^{\mathrm{UK}}$ ) were also observed. Given that mastery of specific IEP behaviours might increase a child's raw score on a particular subtest by several points at best, it is likely that the package of IEP goals focused on during the intervention period contributed to increased CELF-P $\mathrm{PK}^{\mathrm{UK}}$ standard scores. The sample size of the present study did not allow for a finer-grained analysis of intervention effects in particular sub-groups of children, but judging from the results of the Boyle et al. (2007) trial, it is possible that children with different profiles of speech and language difficulties (e.g., those with expressive language delay versus those with mixed receptive-expressive delays) responded to the intervention differently. This possibility should be explored in subsequent studies, since it is also possible that there may not be a differential effect, as the children in the Boyle et al. 
study were older (6-11 years) than those in the present study (4-6 years). The results of the standardized speech assessment (DEAP Percent of Phonemes Correct) showed a significant change in raw scores but not in standard scores (Table 3).

Information from the completed questionnaires involved seeking a range of subjective views from parents/carers and teachers before and after the intervention period. Statistically significant changes were noted for two of 19 items on the parent/carer questionnaire and 10 of 20 items on the teacher questionnaire. The finding that both parents/carers and school staff reported positive changes in how the children were affected by their speech and language difficulties at home and in school subsequent to the intervention, adds credence to the benefit of the input, although since parents of children not receiving the intervention were not asked to complete the questionnaires, the possibility of a Hawthorne effect exists. The positive changes reported on the questionnaires may also reflect progress due to maturation, increased understanding of the child's needs and how to meet these, and/or the importance of partnership working to achieve success. Notwithstanding these possibilities, the questionnaire findings provide valuable information regarding how parents/carers and school staff viewed this intensive input.

It is difficult to make direct comparisons between the present study and previous studies investigating the efficacy or effectiveness of consultative-based approaches because of differences in study methodologies. In an RCT, Boyle et al. (2007) reported significant improvements in standardized test scores regardless of whether intervention was conducted by SLTs or SLTAs (i.e., direct versus indirect approaches). However, in a cohort study reported by McCartney et al. (in press), when a similar intervention was implemented by education staff, children's scores on a standardized language test did not improve. The children who took part in these two studies were older (6-11 years) than those who took part in the present study (4-6 years). The present study involved training school staff by the STA 
so that they could act as agents of therapy following the intensive period of intervention delivered by the STA. In this way, the STA both worked individually with the child and trained school staff to deliver intervention.

The combination of direct intervention and collaboration/consultation with school staff is a feature of the exploratory study reported by Adams and Lloyd (2007), although in that study the direct intervention was delivered by a senior SLT. Direct, intensive specialist intervention, together with classroom-based work and the adaptation of the classroom environment resulted in measurable gains in children's pragmatic skills. Whilst the authors stressed that their study was exploratory, it highlights the importance of collaborative practice, an underlying feature of the enhanced consultancy model reported here.

Although these studies all involved an intensive programme of intervention, the amount of therapy given varied. The average of 39 sessions received by children in the present study is comparable to the 38 sessions delivered in the Boyle et al. (2007) study but exceeds the 26 sessions delivered in the cohort study reported by McCartney et al. (in press) and the 20 sessions delivered in the Adams and Lloyd (2007) study. The ideal amount of therapy required for successful outcomes is yet to be determined and is likely to vary by client group (i.e., groups differing in type or severity of speech, language and communication difficulty).

The intervention targets assessed by the primary outcome measure in the current study represented only a small portion of each child's IEP. It is interesting to note that on average, $69 \%$ of each child's IEP targets were achieved, with $57 \%$ of the total number of targets set comprising speech, receptive or expressive language objectives. It is likely that these contributed to the improvements observed on the standardized tests.

Conclusions. This study suggests that intensive intervention, delivered by a specialist teaching assistant in school, under the guidance of a specialist teacher or teacher/therapist, 
was successful in improving language outcomes. On average, each child received 39 sessions of input, each lasting approximately an hour, delivered over a school term. In addition, interaction between the specialist assistant and specialist teacher took place in order to set targets and monitor progress. Written feedback was given to parents on a daily basis and a dialogue was encouraged. This method of service delivery may be relatively costly compared to other methods of service delivery, since each STA involved in the study was only able to work with three children per term. However, this study indicates that significant gains can be made with a service delivery model that may be considered an expensive method of specialist intervention. Whether similar gains could be achieved with a reduced amount of specialist support is an area for further investigation but it must be noted that to compromise on the quantity and kind of support offered may compromise the progress that might be made.

Limitations of the present study. The fact that linguistic behaviours were not randomly assigned to intervention and control conditions and that control behaviours were not reassessed during the maintenance phase, are recognised as study limitations. Future studies would benefit from random assignment of linguistic behaviours to target and control conditions, as well assessing control behaviours during the maintenance phase. The study's findings are also limited by the fact that the standardized tests (CELF-P ${ }^{\mathrm{UK}}$ and DEAP) were not administered to a control group of children. The case that children not receiving this intervention may have made similar gains in standard scores to those who did receive the intervention cannot be ruled out. It was pointed out that not every child in the study was administered the DEAP and the CELF-P ${ }^{\mathrm{UK}}$ Receptive subscale. The reason for this was a practical one: in the clinical setting in which the study took place, the DEAP is normally administered only to children suspected of having disordered speech. While the CELF-P $\mathrm{P}^{\mathrm{UK}}$ Expressive subscale was administered to all children in this study, five children were not administered the Receptive subscale as they were deemed by professionals not to have 
comprehension difficulties. A further limitation of the present study is that assessors were not blinded when administering and scoring the probes, thus raising the possibility of expectation bias. Although those who administered the tests knew the children had taken part in the intervention programme, it was often the case that pre- and post-intervention assessments were undertaken by different people, well practised in carrying out standardized assessments, thus reducing the possibility of examiner bias.

Directions for future research. The outcomes of this exploratory trial suggest that improvements can be made in children's speech and language abilities through the use of an intensive intervention based on a consultancy approach. Subsequent research, involving a phase III RCT (MRC Health Services and Public Health Research Board 2000) is a possible next step in examining the effect of the intervention suggested in the present phase II exploratory trial. This might be powered so as to be able to explore possible differential effects of the enhanced consultancy model on sub-groups of children having different language profiles. Future research is also needed to explore the clinical and cost effectiveness of the intervention, as required by the UK Department of Health/National Health Service and Department for Education and Skills. 


\section{Acknowledgements}

We gratefully acknowledge the staff of the Communication Support Service for help with data collection, Lindsay Pennington for insightful comments on an earlier draft, and the Newcastle Speech and Language Therapy service for permission to adapt the questionnaires used in this study. We also thank the children, parents and school staff who participated in this study, without whom it could not have taken place.

\section{Declaration of interest}

The first two authors are employed as part of the specialist team that conducted this study.

\section{Notes}

${ }^{1}$ The role of an STA corresponds to that of a Speech and Language Therapy Assistant. Essential qualification for employment is completion of accredited training in speech, language and communication needs to at least Open College Network Accreditation Level 3 (equating to a GNVQ Advanced or GCSE Advance Level).

${ }^{2}$ Specialist teachers have a postgraduate qualification in child language, either at Diploma or at Masters level.

${ }^{3}$ Where a child's needs have been identified as requiring support this is additional to and different from that normally provided by school to meets those needs, specialist input from external agencies is sought. This is referred to as placing a child on the Special Educational Needs register at School Action Plus.

${ }^{4}$ Income Deprivation Affecting Children, part of the government's Index of Multiple Deprivation, is based on the number of children in households receiving means-tested government benefits.

${ }^{5}$ Interpretation of effect sizes for repeated measures designs is based on Bakeman (2005: $383)$. 


\section{References}

ADAMS, C. AND LLOYD, J., 2007, The effects of speech and language therapy intervention on children with pragmatic language impairments in mainstream school. British Journal of Special Education, 34, 226-233.

BAKEMAN, R., 2005, Recommended effect size statistics for repeated measures designs. Behavior Research Methods, 37, 379-384.

BEERY, K.E. AND BuKTENICA, N.A., 1997, The Beery-Buktenica Developmental Test of Visual-Motor Integration, $5^{\text {th }}$ ed. (Upper Saddle Rive, NJ: Pearson Assessments).

BoEHM, A.E., 2001, Preschool Boehm Test of Basic Concepts, $3^{\text {rd }}$ ed. (London: Psychological Corporation).

Boyle, J., McCartney, E., Forbes, J. \& O’Hare, A., 2007, A randomised controlled trial and economic evaluation of direct versus indirect and individual versus group modes of speech and language therapy for children with primary language impairment. Health Technology Assessment, 11(25).

CIRRIN, F.M. AND GILLAM, R.B., 2008, Language intervention practices for school-age children with spoken language disorders: a systematic review. Language, Speech, and Hearing Services in Schools, 39, S110-S137.

DePartment For Children, Schools And FAmilies, 2008, The Bercow Report: A Review of Services for Children and Young People (0-19) with Speech, Language and Communication Needs. http://www.dcsf.gov.uk/slcnaction/downloads/7771-DCSFBERCOW.PDF (accessed 19 August 2008).

DEPARTMENT FOR EDUCATION AND SKILLS, 2001, Special Educational Needs Code of Practice (Nottingham: DfES).

DePARTMENT FOR EDUCATION AND SKILls, 2003, Every Child Matters (Nottingham: DfES). 
Dodd, B., Hua, Z., Crosby, S., Holm, A. And Ozanne, A., 2002, Diagnostic Evaluation of Articulation and Phonology (London: Psychological Corporation).

GASCOIGNE, M., 2006. Supporting children with speech, language and communication needs within integrated children's services (position paper) (London: Royal College of Speech and Language Therapists Position Paper).

Glogowska, M., Roulstone, S., Enderby, P. And Peters, T.J., 2000. Randomised controlled trial of community based speech and language therapy in preschool children. BMJ, 321, 923-927.

LAW, J. AND CONTI-RAMSDEN, G., 2000, Treating children with speech and language impairments: six hours of therapy is not enough. British Medical Journal, 321, 908909.

LAW, J., GARRETT, Z. AND NYE, C., 2004, The efficacy of treatment for children with developmental speech and language delay/disorder: a meta-analysis. Journal of Speech, Language, and Hearing Research, 47, 924-943.

Law, J., Lindsay, G., Peacey, N., Gascoigne, M., Soloff, N., Radford, J. And Band, S.W., 2002, Consultation as a model for providing speech and language therapy in schools: a panacea or one step too far? Child Language Teaching and Therapy, 18, $145-164$.

Lindsay, G., Desforges, M., Dockrell, J., Law, J., Peacey, N. AND BeEcham, J., 2008, Effective and Efficient Use of Resources in Services for Children and Young People with Speech, Language and Communication Needs, Research Report DCSF-RW053. Nottingham: DCSF.

Lindsay, G., Soloff, N. , Law, J., Band, S., PeAcey, N., Gasciogne, M. And Radford, J., 2002, Speech and language therapy services to education in England and Wales. International Journal of Language and Communication Disorders, 37, 273-288. 
MCCARTNEY, E., ElLIS, S. AND BOYLE, J., in press, The mainstream primary classroom as a language-learning environment for children with severe and persistent language impairment - implications of recent language intervention research. Journal of Research in Special Educational Needs.

MRC HeAlth SERVices AND PUblic HeAlth Research BoARd, 2000, A framework for development and evaluation of RCTs for complex interventions to improve health. http://www.mrc.ac.uk/Utilities/Documentrecord/index.htm?d=MRC003372 (accessed 19 August 2008).

OwENS, R.E., 2001, Language Development, An Introduction, $5^{\text {th }}$ edn, (Boston: Allyn and Bacon).

WIIG, E.H., SECord, W. AND SEMEL, E., 2000, Clinical Evaluation of Language Fundamentals-Preschool $^{U K}$ (London: Psychological Corporation).

Zimmerman, I.L., Steiner, V.G. And Evatt Pond, R., 1997, Preschool Language Scale-3 (UK) (London: Psychological Corporation). 
Table 1. Means and standard deviations for children's age, number of intervention sessions and tests administered before intervention

\begin{tabular}{|c|c|c|c|}
\hline & $\mathrm{N}$ & M & SD \\
\hline Age (months) & 35 & 60.26 & 7.29 \\
\hline Number of intervention sessions & 35 & 39.00 & 11.44 \\
\hline PLS-3 Auditory Comprehension ${ }^{1}$ & 29 & 69.59 & 13.61 \\
\hline PLS-3 Expressive Communication ${ }^{1}$ & 31 & 61.55 & 11.46 \\
\hline DEAP Percent phonemes correct ${ }^{2}$ & 21 & 3.05 & 0.22 \\
\hline $\mathrm{VMI}^{1}$ & 35 & 86.14 & 13.09 \\
\hline \multicolumn{4}{|c|}{${ }^{1}$ Standard score based on population mean $=100$ and $\mathrm{SD}=15}$. \\
\hline \multicolumn{4}{|c|}{${ }^{2}$ Standard score based on population mean $=10$ and $\mathrm{SD}=3}$. \\
\hline \multicolumn{4}{|c|}{ PLS-3, Preschool Language Scale-3 UK (Zimmerman et al. 1997); DEAP, Diagnostic } \\
\hline \multicolumn{4}{|c|}{ Evaluation of Articulation and Phonology (Dodd et al. 2002); VMI, Beery-Buktenica } \\
\hline
\end{tabular}


Table 2. Mean number correct out of 10, standard deviation and number of participants for pre-treatment, post-treatment and maintenance probes for two target and two control behaviours

\begin{tabular}{lcccccccccc}
\hline & \multicolumn{3}{c}{ Pre-treatment } & \multicolumn{3}{c}{ Post-treatment } & & \multicolumn{3}{c}{ Maintenance } \\
& M & SD & $\mathrm{n}$ & $\mathrm{M}$ & SD & $\mathrm{n}$ & $\mathrm{M}$ & SD & $\mathrm{n}$ \\
\hline Target 1 & 0.42 & 0.67 & 35 & 9.17 & 2.05 & 35 & 8.63 & 3.19 & 30 \\
Control 1 & 0.07 & 0.21 & 35 & 1.45 & 2.39 & 35 & -- & -- & -- \\
Target 2 & 0.69 & 1.08 & 33 & 8.13 & 2.98 & 33 & 8.64 & 3.30 & 28 \\
Control 2 & 0.28 & 0.77 & 33 & 1.35 & 2.42 & 33 & -- & -- & -- \\
\hline
\end{tabular}


Table 3. Standard scores on standardized tests administered before and after intervention, with test of mean differences and standardized effect size $\left(\eta_{p}^{2)}\right.$ for each measure

\begin{tabular}{|c|c|c|c|c|c|c|c|c|}
\hline & \multicolumn{2}{|c|}{$\underline{\text { Pre-treatment }}$} & \multicolumn{2}{|c|}{$\underline{\text { Post-treatment }}$} & $\Lambda$ & $\mathrm{F}$ & df & $\eta_{\mathrm{p}}^{2}$ \\
\hline Linguistic concepts $^{2}$ & 4.33 & 2.14 & 4.60 & 2.30 & 0.98 & 0.62 & 1,29 & .02 \\
\hline Basic concepts $^{2}$ & 5.63 & 3.11 & 6.33 & 3.68 & 0.95 & 1.60 & 1,29 & .05 \\
\hline CELF-P $P^{\mathrm{UK}}$ expressive composite ${ }^{1}$ & 69.66 & 8.25 & 71.40 & 10.90 & 0.92 & $3.00 *$ & 1,34 & .08 \\
\hline Formulating labels ${ }^{2}$ & 5.03 & 2.08 & 5.57 & 2.64 & 0.90 & $3.95 *$ & 1,34 & .10 \\
\hline Word structure $^{2}$ & 4.23 & 1.42 & 4.91 & 2.32 & 0.89 & $4.13 *$ & 1,34 & .11 \\
\hline $\begin{array}{l}* p<0.05, * * p<0.001 . \\
{ }^{*} \text { Standard score based on populatio } \\
{ }^{2} \text { Standard score based on populatio } \\
\text { CELF-PK }{ }^{\mathrm{UK}} \text {, Clinical Evaluation of } \mathrm{L} \\
\text { Phonology (Dodd et al. 2002). }\end{array}$ & $\begin{array}{l}100 \\
10 \text { an } \\
\text { Fund }\end{array}$ & 5. & Wiio & ); I & agno & ivolunti & . & d \\
\hline
\end{tabular}




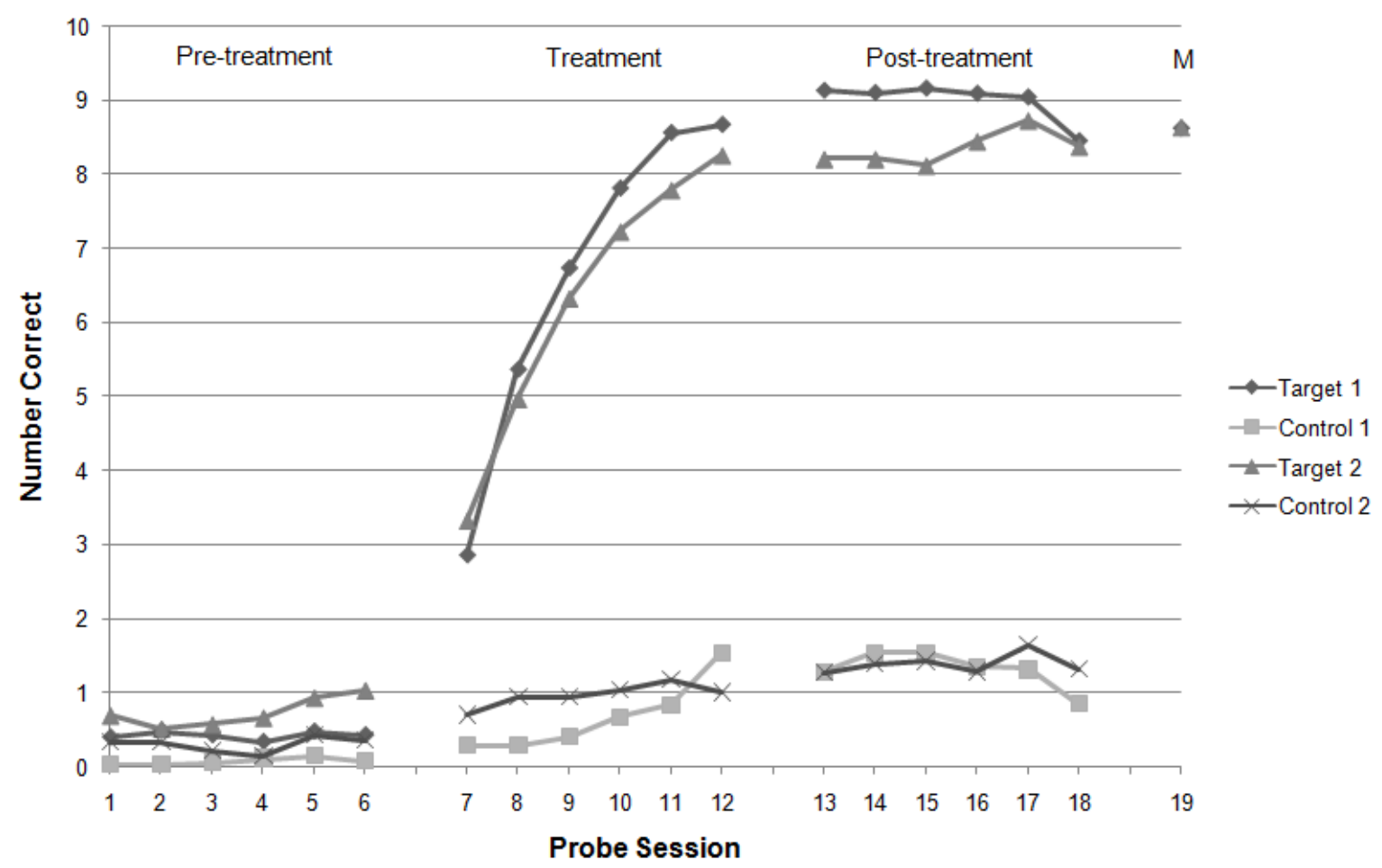

Figure 1. Mean number of items correct on probe task across all children for the two targets and controls during the pre-treatment, treatment, post-treatment and maintenance (M) phases (see Table 2 for sample sizes). Note: the six probes taken during the treatment phase spanned the 10-week intervention period (average of 39 treatment sessions per child). 


\section{Appendix A. Target and control behaviours for each child, with (rec=receptive; exp=expressive target; $\mathrm{WI}=$ =word initial; $\mathrm{WF}=$ =ord final).}

\begin{tabular}{|c|c|c|c|c|}
\hline Participant & Target 1 & Control 1 & Target 2 & Control 2 \\
\hline 1 & he/she (exp) & they (exp) & syllable clapping & $\begin{array}{l}\text { sound } \\
\text { discrimination }\end{array}$ \\
\hline 2 & he/she (exp) & they $(\exp )$ & $\mathrm{WI} / \mathrm{s} /$ & $\mathrm{WI} / \mathrm{f} /$ \\
\hline 3 & /s/ in isolation & $/ \mathrm{w} /$ in isolation & WI /f/ & $\mathrm{WI} / \mathrm{t} /$ \\
\hline 4 & $\mathrm{WI} / \mathrm{g} /$ & $\mathrm{WI} / \mathrm{f} /$ & he/she (exp) & they $(\exp )$ \\
\hline 5 & $/ \mathrm{s} /$ + vowel & /f/ + vowel & sequencing $/ \mathrm{s}, \mathrm{m} /$ & sequencing $/ \mathrm{k}, \mathrm{g} /$ \\
\hline 6 & $\begin{array}{l}\text { behind/in front } \\
\text { (rec) }\end{array}$ & near $(\mathrm{rec})$ & - & - \\
\hline 7 & $/ \mathrm{s} /$ + vowel & 'sh' + vowel & sequencing /p,t,k/ & $\begin{array}{l}\text { sequencing } \\
/ \mathrm{b}, \mathrm{d}, \mathrm{g} /\end{array}$ \\
\hline 8 & he/she (exp) & they (exp) & $/ \mathrm{s} /$ in isolation & $/ \mathrm{f} /$ in isolation \\
\hline 9 & /s/ in isolation & /z/ in isolation & sequencing /p,t,k/ & $\begin{array}{l}\text { sequencing } \\
/ \mathrm{b}, \mathrm{d}, \mathrm{g} /\end{array}$ \\
\hline 10 & $\mathrm{WI} / \mathrm{v} /$ & WF 'ng' & he/she (exp) & they $(\exp )$ \\
\hline 11 & he/she (exp) & they (exp) & in/on/under (exp) & next to/ by (exp) \\
\hline 12 & is+verb+ing & are+verb+ing & $\begin{array}{l}\text { final consonant } \\
\text { deletion }\end{array}$ & $\begin{array}{l}\text { voicing: WF /g/ } \\
\text { to } / \mathrm{k} / \mathrm{WF} / \mathrm{d} / \text { to } \\
/ \mathrm{t} /\end{array}$ \\
\hline 13 & $\mathrm{WI} / \mathrm{s} /$ & WI /f/ & is (aux) & are (aux) \\
\hline 14 & is (aux) & are (aux) & - & - \\
\hline 15 & $\mathrm{WF} / \mathrm{f} /$ & $\mathrm{WI} / \mathrm{s} /$ & is (aux) & are (aux) \\
\hline 16 & $\begin{array}{l}\text { in front/behind } \\
\text { (rec) }\end{array}$ & $\begin{array}{l}\text { next to } \\
(\mathrm{rec})\end{array}$ & is (aux) & are (aux) \\
\hline 17 & $/ \mathrm{s} /+$ vowel & $|z|+$ vowel & $\begin{array}{l}\text { 3WL (no } \\
\text { preposition; exp) }\end{array}$ & $\begin{array}{l}\text { 3WL (incl. prep; } \\
\text { exp) }\end{array}$ \\
\hline 18 & middle (rec) & nearest (rec) & behind (rec) & next to (rec) \\
\hline 19 & $\mathrm{WF} / \mathrm{t} /$ & $\mathrm{WF} / \mathrm{f} /$ & $\mathrm{WI} / \mathrm{s} /$ & WI 'sh' \\
\hline 20 & on $(\exp )$ & under (exp) & plurals & 'and' \\
\hline 21 & he/she (exp) & they (exp) & $\begin{array}{l}\text { final consonant } \\
\text { deletion }\end{array}$ & voicing \\
\hline 22 & he/she (exp) & they (exp) & copula & possessive 's' \\
\hline 23 & he/she (exp) & they (exp) & different (rec) & full (rec) \\
\hline 24 & different (rec) & many (rec) & regular plurals & possessive 's' \\
\hline 25 & same (rec) & bottom (rec) & in/on (rec) & under (rec) \\
\hline 26 & same (rec) & tallest (rec) & on $(\mathrm{rec})$ & in (rec) \\
\hline 27 & $\begin{array}{l}\text { in front/behind } \\
\text { (rec) }\end{array}$ & next to (rec) & he/she (exp) & they $(\exp )$ \\
\hline 28 & $\begin{array}{l}\text { in front/behind } \\
(\exp )\end{array}$ & next to $(\exp )$ & is+veb+ing & are+verb+ing \\
\hline 29 & he/she (exp) & they $(\exp )$ & first last (rec) & different (rec) \\
\hline 30 & he/she (rec) & they (rec) & cluster reduction & deaffrication \\
\hline 31 & different (rec) & many $(\mathrm{rec})$ & he/she (rec) & they (rec) \\
\hline 32 & under (rec) & top (rec) & is+verb+ing & are+verb+ing \\
\hline 33 & his/hers (exp) & theirs $(\exp )$ & first/last (rec) & middle (rec) \\
\hline
\end{tabular}


Effectiveness of an enhanced consultative approach 34

\begin{tabular}{clccc}
\hline Participant & \multicolumn{1}{c}{ Target 1 } & Control 1 & Target 2 & Control 2 \\
\hline 34 & $\begin{array}{l}\text { in front/behind } \\
(\mathrm{rec})\end{array}$ & next to $(\mathrm{rec})$ & different (rec) & bottom (rec) \\
35 & he/she $(\exp )$ & they $(\exp )$ & is+verb+ing & are+verb+ing \\
\hline
\end{tabular}


Appendix B. Questionnaire items. [*] indicates responses to item significantly differed after intervention. Adapted from questionnaires developed by the Newcastle Speech and Language Therapy Service, Newcastle upon Tyne, UK; used with permission.

Parent Questionnaire

1. *Do you think your child's speech and language difficulties affect him/her at home?

2. *Do you think your child's speech and language difficulties affect him/her at school?

3. How would you describe your child?
a. Willing to join in with other children?
b. Willing to talk to familiar adults?
c. Happy to talk to anyone?
d. Confident.
e. Quiet, shy.
f. Frustrated.
g. Preferring to be by himself/herself.
h. Keen to join in new situations.
i. Willing to have 'a go' at new activities.
j. Likes to keep to familiar routines, activities, toys.
k. Likes to keep close to familiar people.
1. Prefers to stay close to you.

4. Does your child enjoy going to school?

5. How clear is your child's speech?

6. Do you feel you receive regular information about your child's progress from school?

7. Do you believe that involvement of the Communication Support Service as a specialist service has increased your understanding of the needs of your child?

8. Do you feel that your child's school has the resources to meet the needs of your child? 


\section{School Questionnaire}

1. *Do you think the pupil's speech and language difficulties affect him/her at school?

2. *How concerned are you about the pupil's progress to date?

3. *How concerned are you about the pupil's future progress?

4. Do you feel the pupil needs additional interventions to enable progress to be made?

5. Do you liaise regularly with parents/carers to provide information about the pupil's progress?

6. Do school staff find it helpful to receive additional specialist information to assist their understanding of the needs of the pupil?

7. *How well does the pupil listen to you?

8. How well does the child follow spoken instructions in class?

9. *How well does the pupil concentrate, attend to a task?

10. How clear is the pupil's speech?

11. How able is the pupil to work independently?

12. How well does the pupil relate to other children?

13. Using the following ratings scale below, can you rate the pupil today in relation to his/her performance in the following areas:
a. *Reading
b. *Writing: letter formation
c. *Writing: sound-letter awareness
d. *Writing: words
e. Writing: sentences
f. *Spelling
g. Numeracy 
14. Is school currently equipped to meet the needs of the pupil? 